\title{
Learning sparse codes from compressed representations with biologically plausible local wiring constraints
}

\author{
Kion Fallah* ${ }^{* \ddagger}$, Adam A. Willats ${ }^{* \ddagger}$, Ninghao Liu ${ }^{\S}$, Christopher J. Rozell ${ }^{\ddagger}$ \\ ${ }^{\ddagger}$ Georgia Institute of Technology, Atlanta, GA, 30332 USA \\ $\S$ Texas A\&M University, College Station, TX, 77843 USA \\ kion@gatech.edu, awillats3@gatech.edu, \\ nhliu43@tamu.edu, crozell@gatech.edu
}

\begin{abstract}
Sparse coding is an important method for unsupervised learning of task-independent features in theoretical neuroscience models of neural coding. While a number of algorithms exist to learn these representations from the statistics of a dataset, they largely ignore the information bottlenecks present in fiber pathways connecting cortical areas. For example, the visual pathway has many fewer neurons transmitting visual information to cortex than the number of photoreceptors. Both empirical and analytic results have recently shown that sparse representations can be learned effectively after performing dimensionality reduction with randomized linear operators, producing latent coefficients that preserve information. Unfortunately, current proposals for sparse coding in the compressed space require a centralized compression process (i.e., dense random matrix) that is biologically unrealistic due to local wiring constraints observed in neural circuits. The main contribution of this paper is to leverage recent results on structured random matrices to propose a theoretical neuroscience model of randomized projections for communication between cortical areas that is consistent with the local wiring constraints observed in neuroanatomy. We show analytically and empirically that unsupervised learning of sparse representations can be performed in the compressed space despite significant local wiring constraints in compression matrices of varying forms (corresponding to different local wiring patterns). Our analysis verifies that even with significant local wiring constraints, the learned representations remain qualitatively similar, have similar quantitative performance in both training and generalization error, and are consistent across many measures with measured macaque V1 receptive fields.
\end{abstract}

\section{Introduction}

Sensory nervous systems have long been championed for their ability to learn effective representations of natural scene statistics [66]. In fact, this ability has been one of the broad motivations behind work in artificial neural networks underlying significant recent advances in machine learning systems. While it is less understood than the supervised learning frameworks common in machine learning, unsupervised learning of task-independent representations is especially important in models of biological sensory systems where supervisory signals are less prevalent. In particular, learning

\footnotetext{
*equal contribution

Code available at: https://github.com/siplab-gt/localized-sparse-coding
} 
a dictionary that admits a sparse latent representation of data (called sparse coding) [49, 50] has become one important theoretical neuroscience model of unsupervised (i.e., self-supervised or autoencoding [33]) learning in sensory systems. Sparse coding models have successfully explained response properties in sensory cortical areas [60, 73, 71, 72, 38, 67, 12] with biologically plausible implementations [60, 63, 73], as well as playing an important role in machine learning algorithms [1, 25, 46, 55, 45, 9, 57, 58.

While a number of detailed models have been proposed to learn such representations from natural scene statistics in a sparse coding framework (with varying levels of biological plausibility) [49. [73, 60], these models of sensory cortices largely ignore the fact that cortical regions are generally connected by very limited fiber projections. Specifically, there are often information bottlenecks where fewer fibers connect cortical areas than the number of neurons encoding the representation in each area [65], meaning that plausible learning algorithms must account for the fact that the sensory data has undergone some type of compression. For example, despite the original formulation of sparse coding for images nominally constituting a model of neural coding in primary visual cortex [49, 51], the pathway carrying that information from retina to cortex (via the lateral geniculate nucleus of the thalamus) has already undergone a significant reduction in the number of neurons carrying visual information transduced by the retinal photoreceptors [68].

Recently, a theoretical model of these information bottlenecks has been proposed based on randomized linear dimensionality reduction (sometimes called compressed sensing [3]) [35, 16, 32]. In this approach, the sparse coding model is learned in the compressed space, producing latent coefficients that capture the same information as if learning had been performed on the raw data. While this approach is counterintuitive because the learned dictionary has randomized structure when inspected, the key insight is that the receptive fields estimated from this model still resemble primary visual cortex (V1) simple cell receptive fields [35, 16]. Randomized dimensionality reduction has appeal as a neural modeling framework for several reasons [5, 26], including the fact that exact wiring patterns between areas are unlikely to be genetically defined [70] and fiber projections can be specified in this framework with coarse chemical gradients to guide randomized fiber growth during development.

Unfortunately, the current proposals for sparse coding in the compressed space [35, 16] are biologically implausible because the compression operator used is a dense random matrix that violates local wiring constraints observed in many neural pathways. For example, in the early visual pathway, a dense compression matrix corresponds to each retinal ganglion cell (RGC) receiving input from every photoreceptor despite neuroanatomical evidence [68] that RGCs receive inputs from a localized region in visual space. It is currently unknown if a randomized linear dimensionality reduction model for fiber projections in neural systems is feasible under biologically plausible local wiring constraints.

The main contribution of this paper is to leverage recent results on structured random matrices to propose a theoretical neuroscience model of randomized projections for communication between cortical areas that is consistent with the local wiring constraints observed in neuroanatomy. We show that unsupervised learning of sparse representations can be performed in the compressed space despite significant local wiring constraints in compression matrices of varying forms (corresponding to different local wiring patterns). Specifically, we provide both an analytic guarantee on the ability to infer unique sparse representations in this model as well as empirical studies on natural images. Our analysis verifies that even with significant local wiring constraints, the learned representations remain qualitatively similar, have similar quantitative performance in both training and generalization error, and are consistent across many measures with measured macaque V1 receptive fields. Taken together, these results constitute one of the few applications of compressed sensing theory to a specific neural coding model [26], showing that biologically plausible randomized encodings can play a significant role in learning structured representations that capture complex natural image statistics.

\section{Background and Related Work}

\subsection{Sparse coding}

In conventional sparse coding (SC), unlabeled training data is used to learn a representation of the statistics of a dataset through a combination of inference (i.e., estimating the latent variables for a data point in a fixed representation) and learning (i.e., adapting the representation). Concretely, assume that a vector $x \in \mathbb{R}^{N}$ is k-sparse in an $N \times P$ dictionary matrix $\Psi$ such that $x=\Psi a$ where 

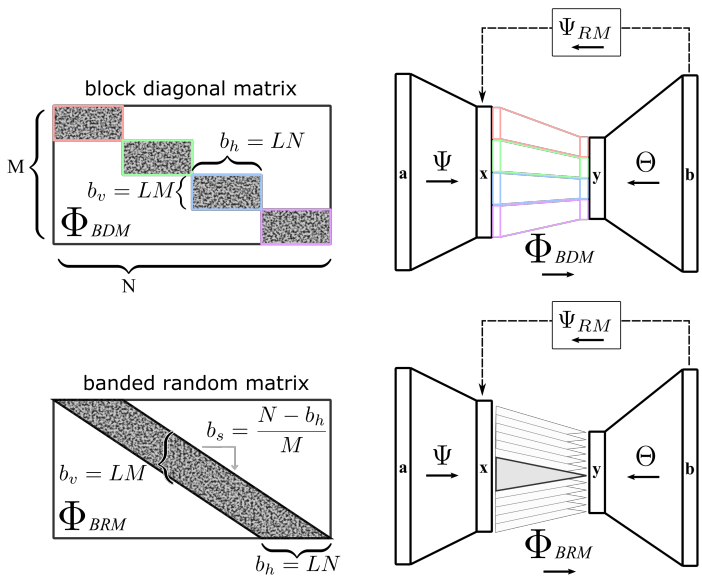

Figure 1: Models of sparse coding on compressed data with with local wiring constraints. We consider two models captured in $M \times N$ random matrices with localization factor $L$ : block diagonal matrices (BDM) and banded random matrices (BRM). Sparse coding is performed on compressed data to learn the dictionary $\Theta$ and the recovered matrix $\Psi_{R M}$ is captured by correlating the coefficients $b$ with the inputs (akin to receptive field estimation). These models reflect the information bottleneck of the early visual pathway, where retinal ganglion cells receive input from a localized set of photoreceptors (adapted from [6]).

$a$ has at most $k$ nonzero entries 2 We follow a common and simple iterative approach to sparse coding [49, 50] using $\ell_{1}$ regularization that defines an energy function:

$$
E(x, \Psi, a)=\|x-\Psi a\|_{2}^{2}+\lambda\|a\|_{1} .
$$

Given a fixed dictionary $\Psi$, inference for a given datapoint $x$ proceeds by minimizing this energy function with respect to $a$ to get the coefficient estimate $\widehat{a}$ and data estimate $\widehat{x}=\Psi \widehat{a}$. Given inferred coefficients for a batch of datapoints, learning is performed by taking a gradient descent step on this energy function with respect to $\Psi$. While SC constitutes a non-convex joint optimization, in practice this approach often converges to reasonable solutions in many natural signal modalities and recent theoretical work has begun to provide recovery guarantees [30, 36, 28, 56, 32, 27]

\subsection{Randomized linear dimensionality reduction}

While there are many approaches to data compression, we concentrate here on randomized linear dimensionality reduction approaches (often appearing in the literature as compressed sensing or compressive sensing [21, 3, 62, 10, 11]) due to the simplicity of biophysical implementation and strong analytic guarantees. This is an established precedent, where previous work with sparse measurement matrices [53] and frame theory [39, 40] have been applied to the recovery of sparse representations of a signal. In these cases, instead of observing $\mathbf{x}$ directly we get the output $\mathbf{y} \in \mathbb{R}^{M}$ after applying a randomized compression matrix $\Phi$ of size $M \times N: \mathbf{y}=\Phi \Psi \mathbf{a}+\mathbf{w}$ where $\mathbf{w}$ represents Gaussian noise. To recover the sparse representation of the original signal, we solve a similar $\ell_{1}$ minimization:

$$
\widehat{\mathbf{a}}(\mathbf{y})=\arg \min _{\mathbf{a}}\|\mathbf{y}-\Phi \Psi \mathbf{a}\|_{2}^{2}+\lambda\|\mathbf{a}\|_{1} .
$$

Despite the challenging nature of the above problem, analytic guarantees can be made on the recovery. For example, when the matrix $\Phi \Psi$ has properties guaranteeing preservation of information in $k$ sparse signals (e.g., near isometries for signal families, etc.), strong asymptotic recovery guarantees can be made [10]. While it is difficult to deterministically specify or verify matrices that satisfy the necessary conditions for recovery, one can typically prove that $\Phi$ matrices generated randomly from many independent distributions (e.g., Gaussian, Bernoulli) work with high probability for any dictionary $\Psi$ when $M=O(k \log N)$ [59]. These extremely favorable scaling laws and universality properties (i.e., working with any $\Psi$ ) are due to such independent dense random matrices having

\footnotetext{
${ }^{2}$ Many results in the literature generalize gracefully in the case that data is only approximately $k$-sparse.
} 
many degrees of freedom and global data aggregation (i.e., every element of $\mathbf{x}$ is used to form each element of $\mathbf{y}$ ). In cases where the matrix $\Phi$ is structured, such recovery guarantees often require slightly more measurements (i.e., polylog in $N$ ) and they are no longer universal because the performance changes depending on $\Psi$ [41, 31, 2, 64]. Of special interest for this paper are recent results detailing the properties of random Block Diagonal Matrices (BDM) [52, 23] and Banded Random Matrices (BRM) [13], which are structured sparse matrices requiring only local data aggregation for compression. In the context of sparse coding, this means that the codes inferred for signals compressed with certain matrices will almost surely be equivalent to codes inferred for the uncompressed signals. Recent research has proposed a dynamical systems model for the visual pathway based on these types of random linear projections, but not in the context of learned sensory representations [5].

\subsection{Dictionary learning from compressed data}

In standard compressed sensing formulations, the matrices $\Phi$ and $\Psi$ are considered known. When the dictionary $\Psi$ is unknown, recovering it exactly from compressed measurements is in general an ill-posed problem known as blind compressed sensing [29]. In the case of interest for this paper, the primary concern is the recovery of latent variables $a$ for use in inference tasks and the matrices $\Psi$ and $\Phi$ are both unknown to the downstream cortical area receiving the compressed data.

Previous work [35, 16] proposed that the sparse representations be learned by performing dictionary learning directly on the compressed data (see Figure 1 . Specifically, the energy function is given by

$$
E(y, \Theta, \mathbf{b})=\|\mathbf{y}-\Theta \mathbf{b}\|_{2}^{2}+\lambda\|\mathbf{b}\|_{1},
$$

where $\Theta=\Phi \Psi$. In this approach, dictionary learning directly on $\mathbf{y}$ is performed according to the alternating minimization procedure outlined earlier by first inferring $\mathbf{b}$ and then taking gradient descent steps with respect to $\Theta$. Recent analytic results [32] have also guaranteed that the optimal representation of the data can be unique (up to a permutation and a scaling).

While SC in the data space produces dictionary elements that visually resemble V1 receptive fields [49. [50], $\Theta$ appears to have random structure after learning because it is learned in the compressed space. While this initially may appear to conflict with observed biology, the key insight of previous work [35, 16] is that electrophysiology experiments do not measure the synaptic wiring patterns (corresponding to the dictionary elements) but rather measures receptive fields corresponding the the stimulus that most activates a neuron. Using a randomized representation (encoded in synaptic weights) does not preclude structured receptive fields as observed in biology. Indeed, by cross correlating the coefficients $\mathbf{b}$ to the original inputs $\mathbf{x}$ (akin to electrophysiologists finding the receptive field of a cell; see Figure 11, previous work shows that it is possible to recover the dictionary $\Psi$ with familiar Gabor wavelet structure and use it for reconstructing the data [35, 16]. Specifically, given access to a subset of data where the uncompressed signals are available, a "reconstruction matrix" (RM) is recovered using $\Psi_{R M}=C_{s r} C_{r r}^{-1}$, where $C_{s r}$ is the cross-correlation matrix $\left\langle\mathbf{x b}^{T}\right\rangle$ and $C_{r r}$ is $\left\langle\mathbf{b} \mathbf{b}^{T}\right\rangle$. Here \langle\rangle represents the empirical mean with respect to given set of data. As a check to recover the sparse representation of original signal, we can estimate a signal reconstruction $\widehat{\mathbf{x}}=\Psi_{R M} \widehat{\mathbf{b}}$ by solving the original optimization with $\Psi_{R M}$ in place of the dictionary:

$$
\widehat{\mathbf{b}}=\arg \min _{\mathbf{b}}\left\|\mathbf{y}-\Phi \Psi_{R M} \mathbf{b}\right\|_{2}^{2}+\lambda_{t}\|\mathbf{b}\|_{1} .
$$

\section{Learning from compressed data with local wiring}

\subsection{Biologically plausible local wiring constraints in random matrices}

In this paper we propose a sparse coding paradigm where learning is performed after compression with a random matrix having biologically plausible local wiring constraints. Wiring minimization has been proposed as constraint faced generally by neural systems throughout development [14, 15]. Given the prevalence of the visual system as inspiration for sparse coding models, we will focus here on the localized wiring patterns observed in the retina as the basis for our theoretical models.

The retina transforms a high-dimensional visual stimulus to a much lower-dimensional neural representation at the optic nerve, with roughly 150 million photoreceptors mapping down to only 1.5 million optic nerve fibers [4]. After photoreceptor transduction, a variety of cell types (bipolar, 
amacrine and horizontal cells) process inputs to drive the retinal ganglion cells (RGC) that are the retinal outputs [47]. The simplified schematic in 1 demonstrates that the retina accomplishes this information compression primarily through the pooling of information from nearby photoreceptors [20]. Spatially, RGCs respond to a finite region in the visual field due to the localized wiring in the mapping from the photoreceptors. When examining how visual space is represented at the optic nerve, it can be seen that collectively representations in RGCs cover the full visual space, with each RGC partially overlapping with the representations of its neighbors [20]. In summary, retinal anatomy generally shows a spatially localized pooling of inputs from photoreceptors to neural representation at the level of RGCs. (See appendix, section 6.1 for further discussion of the statistics of retinal connectivity.)

Given what is known about retinal anatomy, we will use two different structured random matrices as theoretical models of compression with biologically plausible local wiring. The first is a block diagonal matrix (BDM), composed of independently randomized sub-blocks along the matrix [52, 23]. The BDM represents a group of RGCs receiving input from a group of photoreceptors, capturing local wiring in a structure that admits strong analysis but potentially exaggerating the partitioning of RGCs into subgroups. The second is a banded random matrix (BRM), composed of independently randomized rows with a compact support that shifts (with overlap) on each row [13]. The BRM represents a model where each subsequent RGC receives input from a limited set of photoreceptors that has partial overlap with the photoreceptors driving neighboring RGCs.

Specifically, each compression matrix is $M \times N$ with $M<N$ and will be characterized by a localization parameter $L \in(0,1]$ that describes the fraction of each row that has non-zero entries. Each row of a matrix will have $b_{h}=L N$ non-zero entries. In a BDM, the matrix is composed of $(1 / L)$ blocks along the diagonal, consisting of $b_{v}=L M$ non-zero entries in each column. In a BRM, each row has the non-zero support set shifted to the right by $b_{s} \in\left[1, b_{h}\right]$ indices relative to the previous row. Up to nominal edge effects, these shifts are constructed so that the last row ends in the lower right of the matrix (or as close as possible), implying that $M b_{s}=\left(N-b_{h}\right)$. Note that the $b_{s}$ parameter is not needed for the BDM. For both matrix types, the number of non-zeros in the matrix is given by $M b_{h}=L M N$ and we take the limit of $L=1$ to be a fully dense matrix. Non-zero entries are drawn from an i.i.d Gaussian distribution of $\mathcal{N}(0,1 / M)$, and this is easily generalized to other sub-Gaussian distributions or normalizations. Note that this model aggregates measurements from across the image (with each measurement formed from a local region), but this is fundamentally different from the common practice of learning a dictionary on individual patches.

Images must be reshaped into a data vector $\mathbf{x}$ in a way that is consistent with the local wiring observed in the retina. For this application, we "unwrap" the 2D image so that nearby neighboring pixels have nearby indices in the 1D x. While there are many possible methods for performing this unwrapping, we use a space-filling curve (the psuedo-Hilbert curve) through the image as the index in $\mathbf{x}$ [37]. As a result, local structure in the compression matrix Phi corresponds approximately to localized regions of 2D image space, much like the spatially localized receptive fields of RGCs. (See appendix section 6.2 for supporting explanation.)

\subsection{Analytic recovery guarantee}

Using recent results on the properties of structured random matrices and recovery conditions for sparse dictionary learning, we can make the following analytic guarantee for when equivalent representations are inferred in the compressed space with a compression process having either of the local wiring structures (BDM or BRM) described above. While the subsequent empirical results will not be limited to specific sparsity dictionaries, for simplicity of exposition, the unified analytic result below is specific to the case where the sparsity dictionary $\Psi$ is an orthonormal Fourier basis (i.e., a standard DFT matrix). This is a reasonable candidate case given the empirical success of JPEG compression, and these results have some extensions to other sparsity bases (as discussed below).

Theorem 1 Let $\Phi$ be constructed as either a BDM or BRM $M \times N$ matrix with localization parameter $L$ as described above and fixed. Let $\Psi$ be a Fourier orthobasis and generate a dataset $y_{i}=\Phi \Psi a_{i}$ with $a_{i}$ chosen randomly to be $k$-sparse with $k>1$ (i.e., uniformly choose the support set of $k$ nonzeros in $b_{i}$ followed by choosing coefficient values uniformly from $\left.(0,1]\right)$ and $i=1, \ldots, D$ for $D=(k+1)\left(\begin{array}{c}N \\ k\end{array}\right)$. If

$$
M \geq C k \log ^{2} k \log ^{2} N
$$




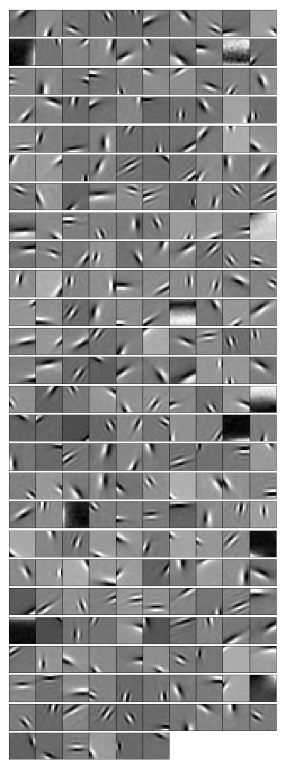

(a) Uncompressed

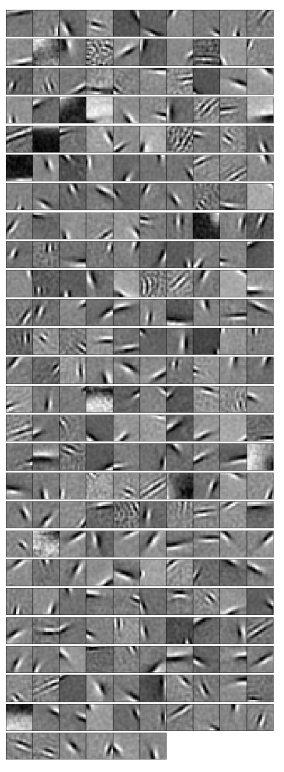

(b) Dense

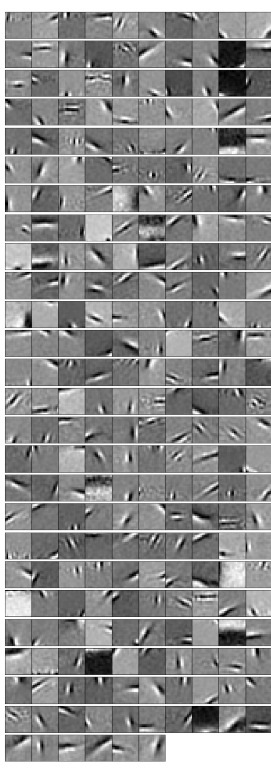

(c) BDM

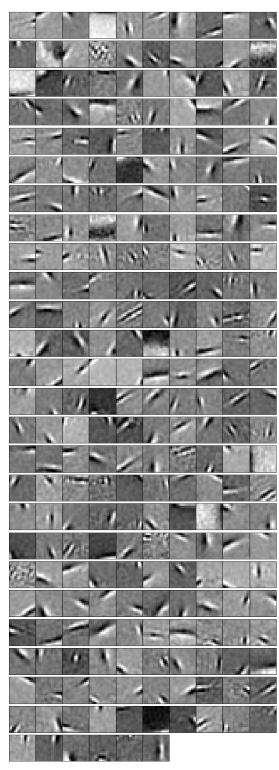

(d) BRM

Figure 2: Recovered dictionaries $\left(\Psi_{R M}\right)$ learned on natural images. (a) Learned on uncompressed patches (conventional SC). (b) Learned after compression with a dense random matrix $(L=1)$. (c) Learned after compression with a block diagonal matrix (BDM) having $L=1 / 16$. (d) Learned after compression with a banded random matrix (BRM) having $L=1 / 16$. Recovered dictionaries are qualitatively similar despite learning after compression under significant local wiring constraints.

for a constant $C$, then with high probability (tending to 1 in the limit of the problem size), any $k$-sparse encoding of the dataset $\left\{y_{i}\right\}$ is equivalent up to an arbitrary permutation and scaling of the columns of $\Phi \Psi$ and the coefficients $\left\{b_{i}\right\}$.

The proof of Theorem 1 is in the appendix, section 6.3. A few comments and observations about this result are warranted. First, note that the above theorem gives conditions for which the ideal coefficients $b$ for the compressed data are equivalent (up to permutation) to the ideal coefficients $a$ for the uncompressed data. This implies that the system will learn the same representations in the sense that their measured receptive fields will also be equivalent (again, up to permutation). While there is no guarantee that realistic numerical algorithms will find these ideal solutions, our empirical results show that they can learn representations that are very close to those learned in the uncompressed case despite having some conditions of the theorem (e.g., orthogonal bases) not strictly met.

Second, note that the statement of the theorem doesn't explicitly depend on the degree of localization $L$ or the matrix type. While the recovery guarantee doesn't depend on $L$, it is possible that the embedding quality (reflected in the isometry constant $\delta$ or scaling constants) could vary with $L$. While we have unified the result for simple exposition, the result is not tight for BRMs [13] and polylog factors could be reduced. Third, while we have presented the results above to keep the exposition as simple as possible, the theorem above could be generalized to characterize the uniqueness and stablilty of the recovery with respect to measurement and modeling error [27].

Finally, given the structured nature of these random matrices, performance will depend on the sparsity basis. In particular, as discussed [52, 23], matrices with localized measurements require a diversity condition that prevents all energy in the image from concentrating in localized blocks. For BDM, Theorem 1 can be extended trivially to any sparsity basis, with performance depending on the incoherence of the basis with the canonical basis (Fourier has optimal incoherence). While general BRM results have not been derived [13], it is likely that similar general results could be shown. 


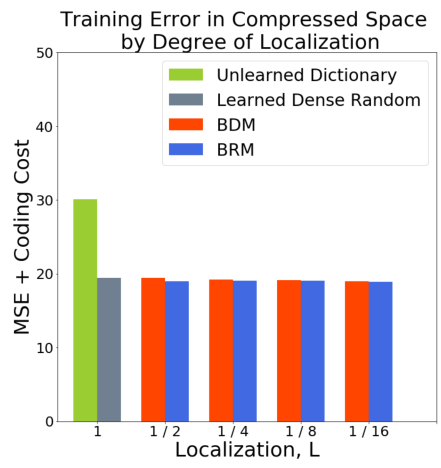

(a)

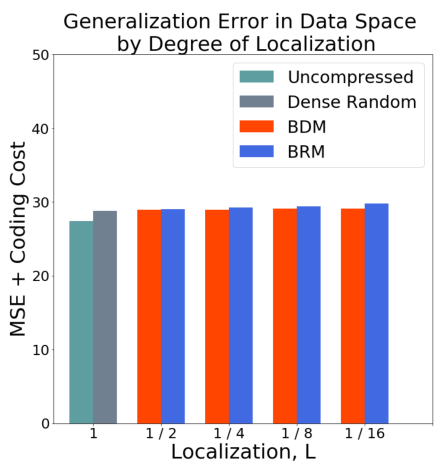

(b)

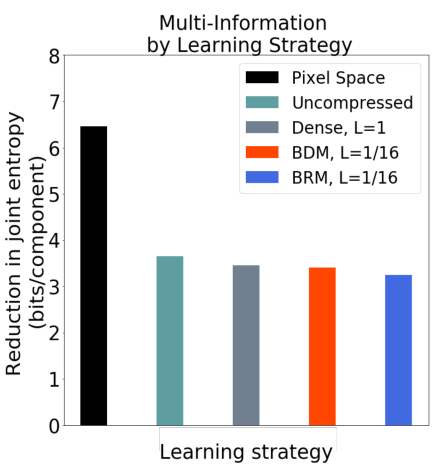

(c)

Figure 3: Coding performance of learned representations. (a) Sparse coding objective (2) for compressed patches using the compressed dictionaries. Unlearned dictionary is equivalent to random initialization before learning, demonstrating that learning improves coding even for randomly compressed data. (b) Generalization error computed with sparse coding objective (1) on patches from hold-out data-set with reconstructed dictionaries $\Psi_{R M}$. (c) Redundancy reduction, as measured through joint entropy, per learning strategy. Uncompressed dictionary is learned with uncompressed patches (i.e. conventional sparse coding). Despite learning with significant compression and wiring constraints, training and generalization is comparable to conventional sparse coding.

\section{Experimental results}

To test the proposed model, we conduct a number of learning experiments using whitened natural image patches compressed with the BDMs and BRMs with varying degrees of localization $(L)$ and compression ratio $M=0.5 \mathrm{~N}$ (other compression ratios did not qualitativly change results, shown in the appendix). Specifically, in our experiments we used 80, $00016 \times 16$ patches extracted from 8 whitened natural images for training. This was broken into batches of size 100, iterated over 150 epochs with decaying step-size on the learning gradient step. To infer coefficients we used $\lambda=5 e-2$, chosen experimentally as a value that produces stable learning convergence. These hyper-parameters were held constant across all experiments. We kept $10 \%$ of the training data-set uncompressed for correlation in recovering $\Psi_{R M}$.

Figure 2 shows the results from learning on natural images with different compression matrices and degrees of localization. This includes uncompressed learning (i.e., conventional SC), and $L=1$, corresponding to the unconstrained compression performed in [35, 16]. The plotted dictionaries for the compressed methods correspond to $\Psi_{R M}$ recovered from correlating uncompressed patches as in [35, 16]. Qualitatively, the recovered dictionaries appear visually similar to conventional SC even with compression and significant levels of localization, demonstrating that biologically plausible local wiring constraints can still yield receptive fields similar to those observed in V1 simple cells.

For quantitative evaluation on the effect of localization, we examine the ability of the learned representations to minimize the coding objectives. This objective is computed in both the compressed space, as well as generalization performance in the (uncompressed) data space. First, Figure 3(a) shows the value of the coding objective in the compressed space (2) evaluated on the training dataset. Figure 3 (b) uses the corresponding recovered dictionaries, $\Psi_{R M}$, to compute the data space objective (1) on a validation data-set for different degrees of localization. The validation data-set was built from 20,000 patches extracted from 2 images not used in training. Note that although these objectives are similar, the compressed space has reduced dimensionality, meaning fewer pixels need to be estimated, thus leading to lower error. Notice that in both measures, there is little change in the objective due to compression or due to the degree of localization. Note also that we plot the training error of an unlearned dictionary, demonstrating that there is significant value to learning the representation even when the data has been subjected to randomized compression. In addition, Figure 3(c) follows previous methods [8, 24] in depicting the redundancy reduction in all learned dictionaries (with small additional reductions when learned in the compressed space, and further small reductions when wiring is localized). 

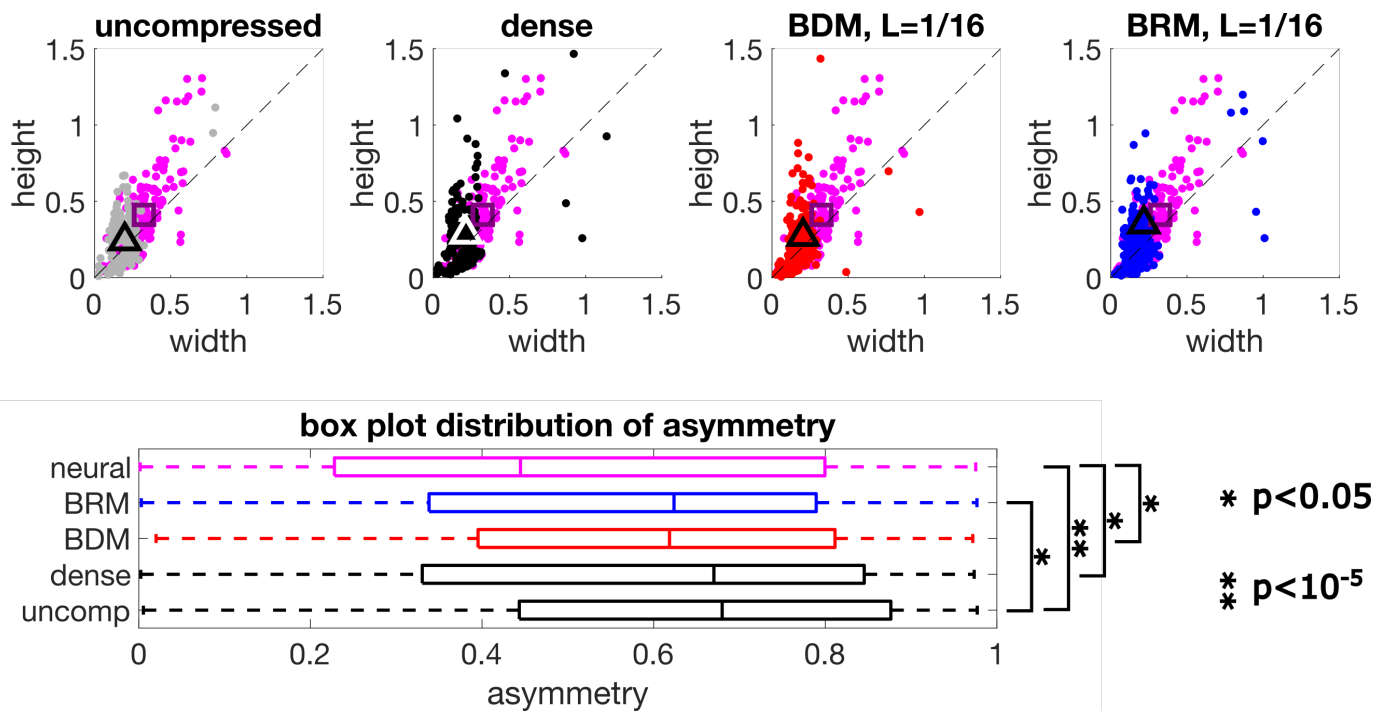

Figure 4: Comparing receptive field characteristics between learned models and macaque V1 measurements [61]. (a, top) This row shows distribution of height and width of gabor wavelet receptive field fits for macaque V1 (magenta) compared to a variety of learned models, including uncompressed, dense matrix $(L=1)$, block diagonal $(L=1 / 16)$ and banded $(L=1 / 16)$. Distribution centroids are marked with triangles for dictionary learning and squares for centroid of V1 neural data. Learning in the compressed space results in receptive field shapes which are qualitatively similar to observed neural data, even for high degrees of localization $(\mathrm{L}=1 / 16)$. ( $b$, below) Box-plot representation of distribution of asymmetry wherein the boundaries of the box represent 25th and 75th percentile of the data as well as the median. Receptive field asymmetry for BRM dictionaries is significantly lower than that of uncompressed matrices $(P<0.05)$, and asymmetry measured from neural data is significantly lower than the uncompressed, dense and BDM models $(P<0.05)$.

Finally, we evaluate the recovered representation by comparing the statistical distribution of learned receptive field shapes to those recovered from electrophysiology experiments in macaque V1 [61]. Following [60] and similar to [61], we fit each recovered dictionary element in $\Psi_{R M}$ with a 2D Gabor function $A \exp \left[-\left(\frac{u^{\prime}}{\sqrt{2} \sigma_{u^{\prime}}}\right)^{2}-\left(\frac{v^{\prime}}{\sqrt{2} \sigma_{v^{\prime}}}\right)^{2}\right] \cos \left(2 \pi f u^{\prime}+\phi\right)$, where $u^{\prime}$ and $v^{\prime}$ are transformed image coordinates, widths of the Gaussian envelopes are given by $\sigma_{u^{\prime}}$ and $\sigma_{v^{\prime}}$, and $f$ is the spatial frequency [22]. Note that envelope width is given by $W=\sigma_{u^{\prime}} f$ and envelope height (referred to as envelope length in [60]) is given by $H=\sigma_{v^{\prime}} f$, measuring the extent of the element relative to the number of spatial frequency cycles it spans. Asymmetry is calculated via the normalized difference of the integral on each side of the $v^{\prime}$ midline as in [60]. Eccentricity, defined as $\left.\mid \log (H / W)\right) \mid$, measures the aspect ratio of the Gabor envelope. Eccentricity is 0 for circularly symmetric receptive fields and larger for more elongated elliptical receptive fields.

Figure 4 quantitatively compares the statistics of the learned dictionary elements. Data from [61], (magenta points in figure 4(a)) show that neural receptive fields cover a wide range of shapes. Previously, it was shown that sparse coding produces a better match than Independent Component Analysis (ICA) [7] to observed cortical receptive fields in macaque V1 [61]. Here we see that a similar distribution of receptive fields characteristics (broadly matching experimentally observed receptive fields) are recapitulated during dictionary learning with a variety of compression strategies, including the significant local wiring constraints of our biologically plausible model.

While the distribution of receptive field widths and heights recovered using the approaches presented here are generally smaller than those recovered from neural data (see also appendix figure 9), a precise match to receptive field size was not a primary goal of this work. A better match to observed neural data might be achievable with a more overcomplete dictionary or by incorporating a nonlinear output stage. 
Due to the importance of asymmetry, used to distinguish learned representations in past research [61], we performed Kruskal-Wallis analysis of variance on the asymmetry to assess whether the learned representations shown in figure 4 (a) all come from the same distribution. We found evidence to reject this null hypothesis with $P<10^{-5}$. However, with further multiple comparisons tests, we saw that receptive field asymmetry for BRM was significantly lower than receptive fields recovered with uncompressed matrices (Figure 4 (b), $P<0.05$ ). Moreover, receptive fields recovered from V1 electrophysiology data were less asymmetric (Figure 4(b)), than those recovered with each method other than BRM (each $P<0.05$ ). Taken together, these results demonstrate that the proposed model learns representations that fit experimental data at least as well as the conventional SC model, with the BRM model potentially fitting experimental data better than learning with uncompressed matrices.

\section{Conclusions}

We have shown for the first time that unsupervised learning of sparse representations can be performed on data compressed using random matrices with biologically plausible local wiring constraints. Using a variety of structure random matrix models, we provide an analytic recovery guarantee as well as empirical characterization showing that the proposed model learns a representation that is at least as good of a fit to visual cortex electrophysiology data as the conventional sparse coding model. These results lead us to conclude that randomized compression with biologically plausible local wiring constraints is a compelling model for information bottlenecks in fiber projections, and the statistics of natural images are highly amenable to unsupervised learning in the proposed model. 


\section{Broader Impact}

This paper presents a theoretical neuroscience model that aims to help improve our understanding of neural coding in biological sensory systems. While this may eventually have secondary effects as inspiration for machine learning systems, the current result is general and theoretical enough that we do not foresee particular applications or negative societal consequences. The images used in the experiments of this paper were the same as those used in previous work on sparse coding [50].

\section{Acknowledgments and Disclosure of Funding}

This work is partially supported by NSF CAREER award CCF-1350954.

\section{References}

[1] M. Aharon, M. Elad, A. Bruckstein, and Y. Katz. K-SVD: An algorithm for designing of overcomplete dictionaries for sparse representations. IEEE Transactions on Signal Processing, 54(11):4311-4322, Oct 2006.

[2] W. U. Bajwa, A. M. Sayeed, and R. Nowak. A restricted isometry property for structurallysubsampled unitary matrices. In 47th Annual Allerton Conference on Communication, Control, and Computing, pages 1005-1012, 2009.

[3] R. G. Baraniuk. Compressive sensing. IEEE Signal Processing Magazine, 24(4), 2007.

[4] HB Barlow. Critical limiting factors in the design of the eye and visual cortex.(the ferrier lecture, 1980.). Proc. Royal Society of London B [Biol. Sci.], 212:1-34, 1981.

[5] V. J. Barranca, G. Kovačič, D. Zhou, and D. Cai. Sparsity and compressed coding in sensory systems. PLoS computational biology, 10(8):e1003793, 2014.

[6] Christian Behrens, Timm Schubert, Silke Haverkamp, Thomas Euler, and Philipp Berens. Connectivity map of bipolar cells and photoreceptors in the mouse retina. Elife, 5:e20041, 2016.

[7] A. J. Bell and T. J. Sejnowski. The "independent components" of natural scenes are edge filters. Vision Research, 37(23):3327-3338, 1997.

[8] Matthias Bethge. Factorial coding of natural images: how effective are linear models in removing higher-order dependencies? J. Opt. Soc. Am. A, 23(6):1253-1268, Jun 2006.

[9] Y. Boureau, F. Bach, Y. LeCun, and J. Ponce. Learning mid-level features for recognition. In 2010 IEEE Computer Society Conference on Computer Vision and Pattern Recognition, pages 2559-2566, 2010.

[10] E. J. Candès. Compressive sampling. In Proceedings of the International Congress of Mathematicians, volume 3, pages 1433-1452. Madrid, Spain, 2006.

[11] E. J. Candès and M. B. Wakin. An introduction to compressive sampling. IEEE Signal Processing Magazine, 25(2):21-30, 2008.

[12] N. L. Carlson, V. L. Ming, and M. R. DeWeese. Sparse codes for speech predict spectrotemporal receptive fields in the inferior colliculus. PLoS Computational Biology, 8(7):e1002594, 2012.

[13] Juan Castorena and Charles D Creusere. The restricted isometry property for banded random matrices. IEEE Transactions on Signal Processing, 62(19):5073-5084, 2014.

[14] Beth L Chen, David H Hall, and Dmitri B Chklovskii. Wiring optimization can relate neuronal structure and function. Proceedings of the National Academy of Sciences, 103(12):4723-4728, 2006.

[15] Dmitri B Chklovskii, Thomas Schikorski, and Charles F Stevens. Wiring optimization in cortical circuits. Neuron, 34(3):341-347, 2002. 
[16] W. K. Coulter, C. J. Hillar, G. Isley, and F. T. Sommer. Adaptive compressed sensing-a new class of self-organizing coding models for neuroscience. In 2010 IEEE International Conference on Acoustics, Speech and Signal Processing, pages 5494-5497, 2010.

[17] Christine A. Curcio and Kimberly A. Allen. Topography of ganglion cells in human retina. Journal of Comparative Neurology, 300(1):5-25, 1990.

[18] Christine A. Curcio, Kenneth R. Sloan, Robert E. Kalina, and Anita E. Hendrickson. Human photoreceptor topography. Journal of Comparative Neurology, 292(4):497-523, 1990.

[19] Jonathan B. Demb and Joshua H Singer. Intrinsic properties and functional circuitry of the AII amacrine cell. Visual neuroscience, 19(1):51-60, 2012.

[20] Steven H Devries and Denis A Baylor. Mosaic arrangement of ganglion cell receptive fields in rabbit retina. Journal of neurophysiology, 78(4):2048-2060, 1997.

[21] D. L. Donoho. Compressed sensing. IEEE Transactions on Information Theory, 52(4):12891306, 2006.

[22] Gerrit Ecke. fit2dGabor, 2020. Library Catalog: www.mathworks.com.

[23] A. Eftekhari, H.L. Yap, C.J. Rozell, and M.B. Wakin. The restricted isometry property for random block diagonal matrices. Applied and Computational Harmonic Analysis, 38(1):1-31, January 2015.

[24] Jan Eichhorn, Fabian Sinz, and Matthias Bethge. Natural Image Coding in V1: How Much Use Is Orientation Selectivity? PLOS Computational Biology, 5(4):1-16, 2009. Publisher: Public Library of Science.

[25] M. Elad, M.A.T. Figueiredo, and Y. Ma. On the role of sparse and redundant representations in image processing. IEEE Proceedings - Special Issue on Applications of Compressive Sensing \& Sparse Representation, Oct 2008.

[26] S. Ganguli and H. Sompolinsky. Compressed sensing, sparsity, and dimensionality in neuronal information processing and data analysis. Annual Review of Neuroscience, 35:485-508, 2012.

[27] Charles J Garfinkle and Christopher J Hillar. On the uniqueness and stability of dictionaries for sparse representation of noisy signals. IEEE Transactions on Signal Processing, 67(23):58845892, 2019.

[28] Q. Geng and J. Wright. On the local correctness of 11-minimization for dictionary learning. In 2014 IEEE International Symposium on Information Theory, pages 3180-3184. IEEE, 2014.

[29] S. Gleichman and Y. C. Eldar. Blind compressed sensing. IEEE Transactions on Information Theory, 57(10):6958-6975, 2011.

[30] R. Gribonval and K. Schnass. Dictionary identification-sparse matrix-factorization via 11minimization. IEEE Transactions on Information Theory, 56(7):3523-3539, 2010.

[31] J. Haupt, W. U. Bajwa, G. Raz, and R. Nowak. Toeplitz compressed sensing matrices with applications to sparse channel estimation. IEEE Transactions on Information Theory, 56(11):5862$5875,2010$.

[32] C. J. Hillar and F. T. Sommer. When can dictionary learning uniquely recover sparse data from subsamples? IEEE Transactions on Information Theory, 61(11):6290-6297, 2015.

[33] G. E. Hinton. Connectionist learning procedures. In Machine learning, pages 555-610. Elsevier, 1990.

[34] Yosef Hochberg and Ajit C Tamhane. Multiple comparison procedures. John Wiley \& Sons, Inc., 1987.

[35] G. Isely, C. Hillar, and F. Sommer. Deciphering subsampled data: adaptive compressive sampling as a principle of brain communication. In Advances in neural information processing systems, pages 910-918, 2010. 
[36] R. Jenatton, R. Gribonval, and F. Bach. Local stability and robustness of sparse dictionary learning in the presence of noise. arXiv preprint arXiv:1210.0685, 2012.

[37] Kim Kenny. hilbertCurve, 2020. Library Catalog: www.mathworks.com.

[38] P. D. King, J. Zylberberg, and M. R. DeWeese. Inhibitory interneurons decorrelate excitatory cells to drive sparse code formation in a spiking model of V1. Journal of Neuroscience, 33(13):5475-5485, 2013.

[39] J. Kovacevic and A. Chebira. Life beyond bases: The advent of frames (part i). IEEE Signal Processing Magazine, 24(4):86-104, 2007.

[40] J. Kovacevic and A. Chebira. Life beyond bases: The advent of frames (part ii). IEEE Signal Processing Magazine, 24(5):115-125, 2007.

[41] F. Krahmer, S. Mendelson, and H. Rauhut. Suprema of chaos processes and the restricted isometry property. arXiv preprint arXiv:1207.0235, 2012.

[42] Sammy C.S. Lee, Paul R. Martin, and Ulrike Grünert. Topography of neurons in the rod pathway of human retina. Investigative Ophthalmology and Visual Science, 60(8):2848-2859, 2019.

[43] Jan Yie Liang, Chih Sheng Chen, Chua Huang Huang, and Li Liu. Lossless compression of medical images using Hilbert space-filling curves. Computerized Medical Imaging and Graphics, 32(3):174-182, 2008.

[44] Zhuolin Liu, Kazuhiro Kurokawa, Furu Zhang, John J. Lee, and Donald T. Miller. Imaging and quantifying ganglion cells and other transparent neurons in the living human retina. Proceedings of the National Academy of Sciences of the United States of America, 114(48):12803-12808, 2017.

[45] J. Mairal, F. Bach, J. Ponce, G. Sapiro, and A. Zisserman. Discriminative learned dictionaries for local image analysis. In IEEE Conference on Computer Vision and Pattern Recognition, 2008.

[46] J. Mairal, G. Sapiro, and M. Elad. Learning multiscale sparse representations for image and video restoration. Multiscale Modeling \& Simulation, 7(1):214-241, 2008.

[47] Richard H Masland. The neuronal organization of the retina. Neuron, 76(2):266-280, 2012.

[48] Bongki Moon, H. V. Jagadish, Christos Faloutsos, and Joel H. Saltz. Analysis of the clustering properties of the Hilbert space-filling curve. IEEE Transactions on Knowledge and Data Engineering, 13(1):124-141, 2001.

[49] B. A. Olshausen and D. Field. Emergence of simple-cell receptive field properties by learning a sparse code for natural images. Nature, 381(13):607-609, Jun 1996.

[50] B. A. Olshausen and D. Field. Sparse coding with an overcomplete basis set: A strategy employed by V1? Vision Research, 37(23):3311-3325, 1997.

[51] B. A. Olshausen and D. J. Field. Sparse coding of sensory inputs. Current Opinion in Neurobiology, 14:481-487, 2004.

[52] J.Y. Park, H.L. Yap, C.J. Rozell, and M.B. Wakin. Concentration of measure for block diagonal matrices with applications to compressive signal processing. IEEE Transactions on Signal Processing, 59(12):5859-5875, December 2011.

[53] F. Parvaresh, H. Vikalo, S. Misra, and B. Hassibi. Recovering sparse signals using sparse measurement matrices in compressed dna microarrays. IEEE Journal of Selected Topics in Signal Processing, 2(3):275-285, 2008.

[54] Christian S Perone. Google's S2, geometry on the sphere, cells and Hilbert curve, 2015.

[55] R. Raina, A. Battle, H. Lee, B. Packer, and A. Y. Ng. Self-taught learning: transfer learning from unlabeled data. In Proceedings of the International Conference on Machine learning, pages 759-766. ACM, 2007. 
[56] S. Rambhatla, X. Li, and J. Haupt. Noodl: Provable online dictionary learning and sparse coding. In International Conference on Learning Representations, 2019.

[57] M. Ranzato, Y. Boureau, and Y. LeCun. Sparse feature learning for deep belief networks. In Advances in neural information processing systems, pages 1185-1192, 2008.

[58] M. Ranzato, F. Huang, Y. Boureau, and Y. LeCun. Unsupervised learning of invariant feature hierarchies with applications to object recognition. In Proceedings of the Conference on Computer Vision and Pattern Recognition, volume 127, 2007.

[59] H. Rauhut. Compressive sensing and structured random matrices. Theoretical Found. and Numerical Methods for Sparse Recovery, 9:1-92, 2010.

[60] M. Rehn and F. T. Sommer. A network that uses few active neurones to code visual input predicts the diverse shapes of cortical receptive fields. Journal of Computational Neuroscience, 22(2):135-146, 2007.

[61] D. L. Ringach. Spatial structure and symmetry of simple-cell receptive fields in macaque primary visual cortex. Journal of Neurophysiology, 88(1):455-463, 2002.

[62] J. Romberg. Imaging via compressive sampling. IEEE Signal Processing Magazine, 25(2):1420, 2008.

[63] C.J. Rozell, D.H Johnson, R.G. Baraniuk, and B.A. Olshausen. Sparse coding via thresholding and local competition in neural circuits. Neural Computation, 20(10):2526-2563, October 2008.

[64] M. Rudelson and R. Vershynin. On sparse reconstruction from fourier and gaussian measurements. Communications on Pure and Applied Mathematics, 61(8):1025-1045, 2008.

[65] A. Schüz, D. Chaimow, D. Liewald, and M. Dortenman. Quantitative aspects of corticocortical connections: a tracer study in the mouse. Cerebral Cortex, 16(10):1474-1486, 2005.

[66] Eero P Simoncelli and Bruno A Olshausen. Natural image statistics and neural representation. Annual review of neuroscience, 24(1):1193-1216, 2001.

[67] E. C. Smith and M. S. Lewicki. Efficient auditory coding. Nature, 439(7079):978, 2006.

[68] D. C. Van Essen, C. H. Anderson, and D. J. Felleman. Information processing in the primate visual system: an integrated systems perspective. Science, 255(5043):419-423, 1992.

[69] Luiz Velho and Jonas Miranda De Gomes. Digital halftoning with space filling curves. SIGGRAPH, 1991.

[70] R. J. Wyman and J. B. Thomas. What genes are necessary to make an identified synapse? In Cold Spring Harbor symposia on quantitative biology, volume 48, pages 641-652. Cold Spring Harbor Laboratory Press, 1983.

[71] M. Zhu and C.J. Rozell. Visual nonclassical receptive field effects emerge from sparse coding in a dynamical system. PLoS Computational Biology, 9(8):e1003191, August 2013.

[72] M. Zhu and C.J. Rozell. Modeling inhibitory interneurons in efficient sensory coding models. PLoS Computational Biology, 11(7):e1004353, July 2015.

[73] J. Zylberberg, J. T. Murphy, and M. R. DeWeese. A sparse coding model with synaptically local plasticity and spiking neurons can account for the diverse shapes of V1 simple cell receptive fields. PLoS computational biology, 7(10):e1002250, 2011. 


\section{Appendix}

\subsection{Statistics of retinal wiring}

One key goal of this work is to introduce an approach to sparse coding which respects the spatially localized connectivity present in the early visual pathway (from images projected onto the retina, to representations in the retinal ganglion cells). In the visual system, each RGC is connected to a subset of photoreceptors which gather light from a contiguous, roughly circular region of an image termed its receptive field. It has been shown that the receptive fields of individual RGCs partially overlap with those of neighboring RGCs. In summary the connectivity of the visual system is sparse and spatially localized. Our intention is to capture the essence of local wiring in the retina, as such support of each row of $\Phi$ are restricted to a small range. However neither the block/band size nor the degree of overlap are intended to be a direct fit to levels of localization and overlap in the biological retina. Instead we focus on characterizing a range of levels of localization to better understand the theoretical and empirical implications of this approach.

The properties of the retina are highly non-uniform in space, with a central fovea containing a high density of cones $\left(\sim 50 \mathrm{kcells} / \mathrm{mm}^{2}\right)$ and an outer periphery of relatively higher density of rods (at peak, $\sim 150 \mathrm{kcells} / \mathrm{mm}^{2}$ ) [18, 42]. The density of RGCs peaks at an intermediate distance $\left(\sim 30 \mathrm{kcells} / \mathrm{mm}^{2}\right)$ and decreases further out [17, 44]. From this, we can infer that the ratio of photoreceptors to RGCs varies between close to 2:1 or 1:1 near the fovea, up to 100s of rods:1 RGC in the periphery. Including the pooling of photoreceptors introduced by horizontal and amacrine cells each RGC may pool up anywhere from 1 to 1000 nearby photoreceptors [19] depending on cell-type and location in the retina. In summary, retinal anatomy generally shows a spatially localized pooling of inputs from photoreceptors to neural representation at the level of RGCs.

\subsection{Spatially-localized image pre-processing}

As part of the sparse coding approach we transform a $2 \mathrm{D}$ image into a $1 \mathrm{D}$ vector $\mathbf{x}$. In order to emulate the local connectivity constraints of the retina, we would like neighboring pixels in $2 \mathrm{D}$ image-space to map to neighboring pixels in $1 \mathrm{D}$ vector-space. To accomplish this we borrow the Hilbert curve (figure 57, because of its simple construction and locality-preserving property. This step involves generating a space-filling curve which traces a path through the pixels of the image, then assigning indices in the $1 \mathrm{D}$ vector according to their position along the curve [37].

It has been shown that using Hilbert curves, nearby indices in 1D dimension are mapped to nearby pixels in 2D. While the converse is not always guaranteed (that neighboring pixels in 2D must map to neighboring elements of their $1 \mathrm{D}$ vector) it has been shown that the Hilbert curve approach maintains locality better than several alternatives [48]. As such, it has been applied to various applications from image compression [69, 43] to storing geographic coordinates in contiguous segments of a 1D memory address register [54].

Because the vector $\mathbf{x}$ uses a spatially contiguous representation of the image, and because of the local structure present in the compression matrix $\Phi$, local regions of image space also correspond to localized segments of $\mathbf{y}$. This is further illustrated in figure 6 where we show smoothly varying $2 \mathrm{D}$ images map to qualitatively smooth $1 \mathrm{D}$ vectors.

\subsection{Proof of Theorem 1}

Draw a random matrix according to the construction specified in section 3.2 and fix it. Under the conditions of the theorem, we first establish that for either random matrix construction, the combined matrix $\Phi \Psi$ satisfies a Restricted Isometry Property (RIP)

$$
(1-\delta)\|\mathbf{a}\|_{2}^{2} \leq\|\Phi \Psi a\|_{2}^{2} \leq(1+\delta)\|\mathbf{a}\|_{2}^{2}
$$

for all $a$ that are $2 k$-sparse (i.e., $\|a\|_{0} \leq 2 k$ ) with $0 \leq \delta<1$.

First we consider BDM matrices. Theorem 1 in [23] establishes that BDM matrices satisfy the RIP with isometry constant $0<\delta<1$ for $M \geq C k \log ^{2} k \log ^{2} N$ with $C$ that depends on $\delta$ and with probability greater than $O\left(1-N^{-\log N \log ^{2} k}\right)$. Note that Theorem 1 in [23] depends on the incoherence of the sparsity dictionary with the canonical basis (denoted $\tilde{\mu}$ in Theorem 1 of [23]), which is established as $\widetilde{\mu}=1$ for the Fourier basis in [23]. We also observe that this result is 

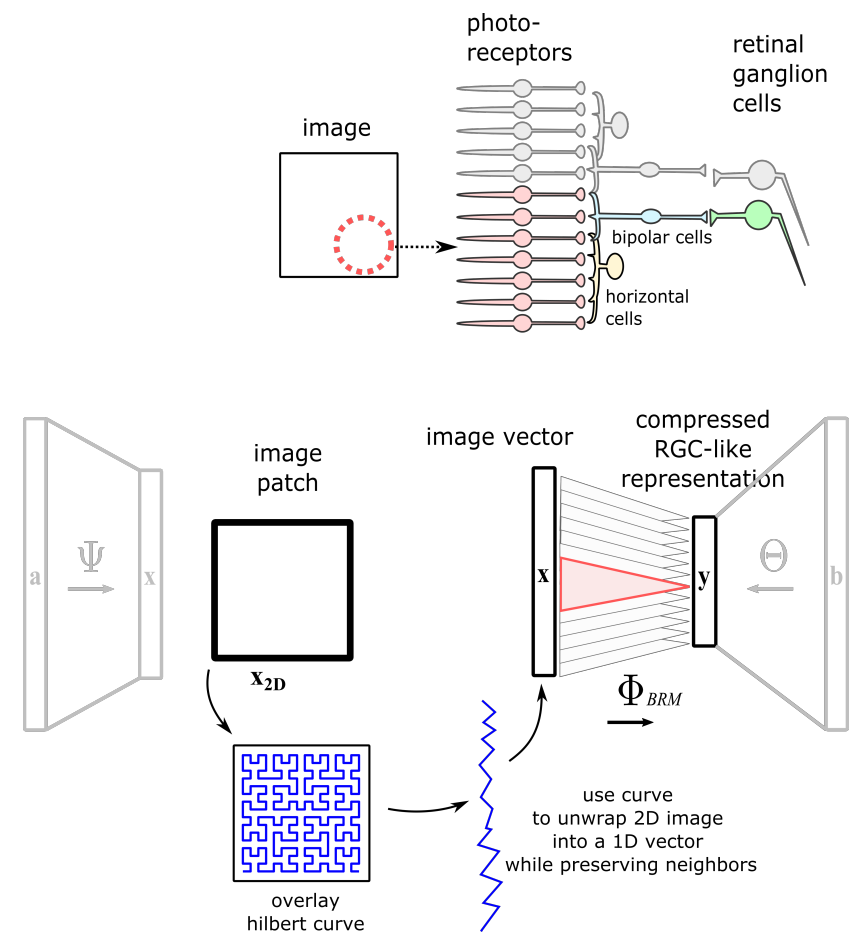

Figure 5: Procedure for preserving locality in models of sparse coding with wiring constraints. While images are represented as values in 2D space, the sparse coding approach presented here operates on $1 \mathrm{D}$ vectors. In order to map pixel locations to vector indices, a Hilbert curve is drawn through the image. Positions along the curve correspond to indices in the vector which can be thought of as unwrapping the curve into a line. By combining this unwrapping with compressed sensing matrices, representations in this method maintain locality from $2 \mathrm{D}$ image space, to the compressed representation.

modifiable in a straightforward way for other sparsity bases with known incoherence. Finally, we also note that the failure probably goes to zero as $N \rightarrow \infty$.

Next we consider BRM martices, with the same discarding of edge effects in the BRM matrix as done in [13]. Theorem 2 in [13] establishes that BRM matrices satisfy the RIP with isometry constant $0<\delta<1$ for $M \geq C k(\log (N / k)+1)$ with $C$ that depends on $\delta$ and with probability greater than $1-2 e^{-C M \delta^{2}}$. Examining these conditions, we see that

$$
\begin{aligned}
k(\log (N / k)+1) & =k(\log (N)-\log (k)+1) \\
& <\tau k\left(\log _{2}(N)\right)<\widetilde{\tau} k\left(\log ^{2}(N) \log ^{2}(k)\right),
\end{aligned}
$$

where the second line follows as long as $k>1$, with $\tau$ and $\widetilde{\tau}$ constants due to a change of log base. The final quantity establishes that the conditions of the present theorem ensure that the conditions of Theorem 2 in [13] hold. While we have presented one unified result for both matrix types, note that this result is not tight for the BRM matrices and a much more aggressive compression (i.e., lower $M$ ) is permissible, but at the expense of generality to other type of sparsity bases. Finally, we also note that the failure probably goes to zero as $M \rightarrow \infty$, which is necessary as $N \rightarrow \infty$.

When RIP holds, for any pair of $k$-sparse vectors $a_{1}$ and $a_{2}, \Phi \Psi\left(a_{1}-a_{2}\right)=0$ implies that $a_{1}=a_{2}$. This can be restated as

$$
\Phi \Psi a_{1}=\Phi \Psi a_{2} \Longrightarrow a_{1}=a_{2},
$$

which is known as the spark condition that characterizes the linear dependence structure of the columns of $\Phi \Psi$. Given the spark condition satisfied for $\Phi \Psi$, Corollary 2 in [32] establishes that with probability one, for $D=(k+1)\left(\begin{array}{l}N \\ k\end{array}\right)$ datapoints drawn randomly (i.e., uniformly choose the support set of nonzeros in $a$ followed by choosing coefficient values uniformly from $(0,1])$, any $k$-sparse encoding of the dataset is equivalent up to an arbitrary permutation and scaling of the columns of $\Phi \Psi$ and coefficients $b_{i}$. 


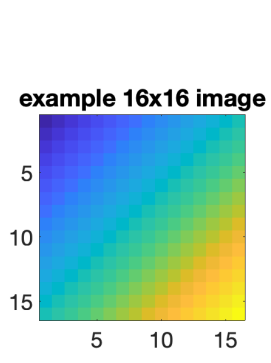

(a)

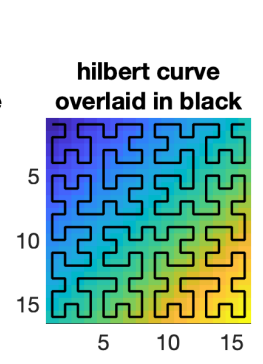

(b)

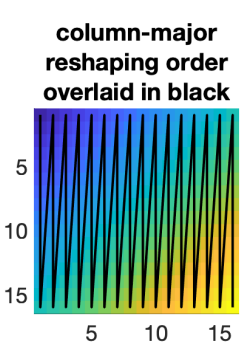

(d)

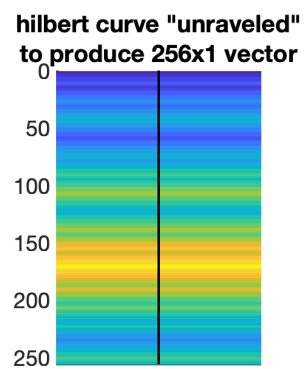

(c)

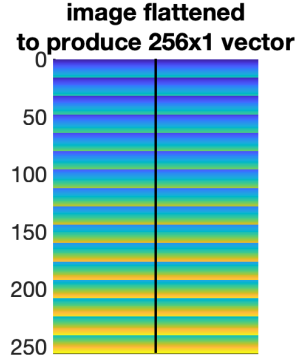

(e)

Figure 6: Illustration of preserved image features after unwrapping. (a) smoothly-varying pixel values for a 16x16 image (b) The Hilbert curve method involves assigning an order to pixels based on the curve's path shown in black. (c) Using the curve's ordering, pixels in 2D space are arranged or unraveled into a $256 \times 1$ vector. Pixel values are seen to relate to their spatial neighbors in a smoothly-varying fashion qualitatively similar to the original 2D image. (d) An alternative approach would be to simply stack the columns to reshape the image. The equivalent path through $2 \mathrm{D}$ space is again visualized in black. (e) As a result of the discontinuities between columns, the image flattened with this approach exhibits periodic jumps in pixel intensity not present in the original 2D image.

\subsection{Result of changing compression}

Additional tests were run to determine the effect of the compression ratio on the generalization error. This error was computed with the sparse coding objective in the data space (as in (1)), with patches not used in training. With a constant localization $L=1 / 4$ for the BDM and BRM matrices, we tested compression ratios of $1 / 2,3 / 8$, and $1 / 4$. The results can be seen in Figure 7, where we see a small increase in generalization error with more compression. Note that as the compression ratio increases, the measurement function has fewer parameters to support gathering information for learning and more of the training data is discarded. It can also be seen that the generalization error is only slightly higher for localized matrices at each compression ratio.

\subsection{Gabor feature quantification}

To understand the characteristics of receptive fields estimated through our technique, we quantified width, height, asymmetry and eccentricity of the receptive fields (see experimental results, figure 4). Figure 8 compares the two dimensional distribution of asymmetry and eccentricity between receptive fields learned through the methods presented here, to those fit to neural visual system data. The distributions cover a similar range across methods.

In figure 9 we show the one-dimensional distribution of each of these features and compare across methods. Width and height occupy a similar range across all conditions except the macaque neural data which has larger Gabor envelopes.

In testing for significant differences in Gabor features across methods, we performed a multiple comparisons test. This extends the Kruskal-Wallis analysis shown in the main text, for which the null hypothesis is that all groups of data come from the same underlying distribution. From this analysis, 


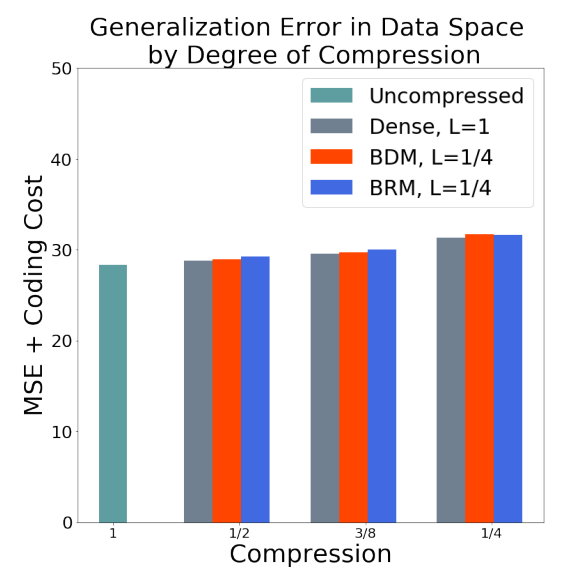

Figure 7: Coding performance of learned representations with varying degrees of compression and localization held constant to $L=1 / 4$. Tested on 20,000 validation patches not used in training.
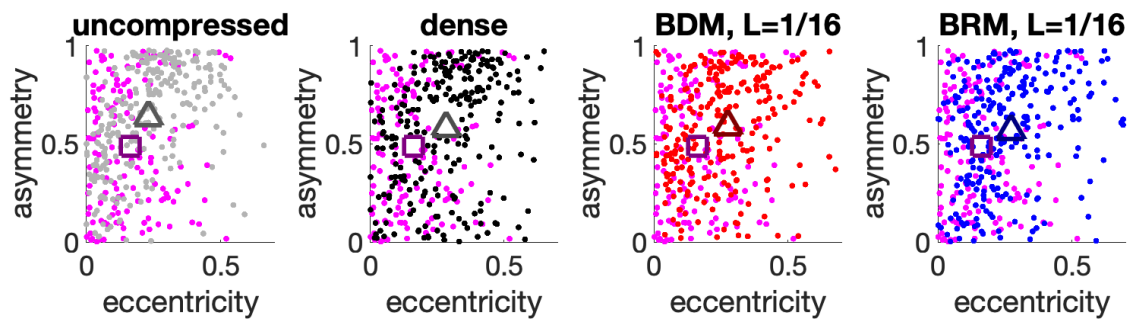

Figure 8: Comparing receptive field asymmetry and eccentricity between learned models and macaque V1 measurements [61]. Asymmetry is calculated from the normalized difference in intensity above and below the midline of the wavelet. Eccentricity is measured as $a b s\left(\log _{10}(H / W)\right)$. Distribution centroids are marked with triangles for dictionary learning and squares for centroid of V1 neural data. Learning in the compressed space results in receptive field shapes which are qualitatively similar to observed neural data, even for high degrees of localization $(\mathrm{L}=1 / 16)$.

we have evidence to reject the null hypothesis at a significance level $\alpha=0.05$ with $P=3.6 * 10^{-6}$. In this multiple comparisons test, we look at pairs of groups (i.e. uncompressed versus BRM) and calculate P-values shown in table 1. For these tests, the null hypothesis is that data from the two groups comes from the same distribution. As such, these P-values represent an upper bound on the probability of falsely identifying significant differences between groups [34].

\begin{tabular}{r|c|c|c|c} 
& dense & BDM & BRM & neural \\
\hline uncompressed & 0.298 & 0.226 & $\mathbf{0 . 0 4 0}$ & $\mathbf{9 . 1 1 e - 0 6}$ \\
\hline dense & & 0.999 & 0.906 & $\mathbf{0 . 0 2 5}$ \\
\hline BDM & $"$ & & 0.951 & $\mathbf{0 . 0 3 8}$ \\
\hline BRM & $"$ & & & 0.221
\end{tabular}

Table 1: P-values from comparing asymmetry across pairs of conditions through Kruskal-Wallis analysis of variance. BDM, BRM are shown for localization $L=1 / 16$. P-values less than a significance level of $\alpha=0.05$ are highlighted in bold. The null hypothesis that the medians of all groups are equal can be rejected with $P=3.6 * 10^{-6}$ 

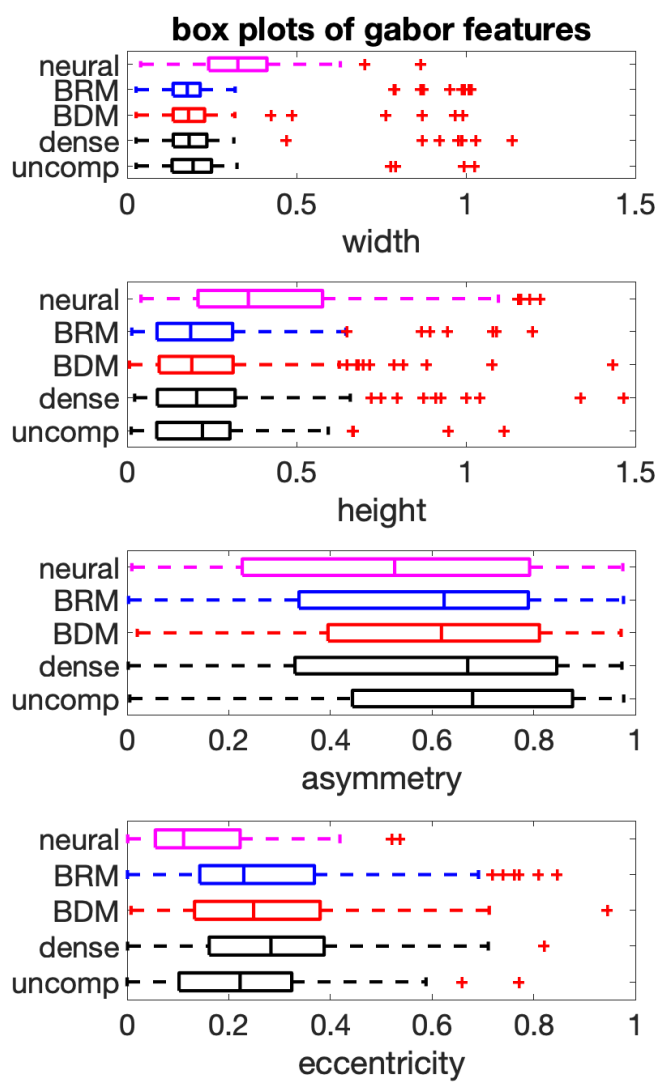

Figure 9: Comparing 1D distribution of receptive field characteristics between learned models and macaque V1 measurements [61]. Box plots shows distributions of Gabor features across conditions. Median, 25th, and 75th percentile shown as box boundaries. BDM, BRM are shown for localization $L=1 / 16$ 